\title{
EFEITO DO ARMAZENAMENTO NA COMPATIBILIDADE DE FUNGICIDAS E INSETICIDAS, ASSOCIADOS OU NÃO A UM POLÍMERO NO TRATAMENTO DE SEMENTES DE FEIJÃO ${ }^{(1)}$
}

\author{
ROSANA GONÇALVES BARROS ${ }^{(2,5)}$; JOSÉ ALEXANDRE F. BARRIGOSSI ${ }^{(3)}$; \\ JEFFERSON LUIS DA SILVA COSTA ${ }^{(4,5)}$
}

\begin{abstract}
RESUMO
Estudou-se neste trabalho a compatibilidade de produtos químicos, associados a um polímero no tratamento de sementes de feijão, estabelecendo-se ainda, o período máximo de armazenamento. Sementes de feijão da cultivar Pérola foram submetidas aos seguintes tratamentos: 1) testemunha; 2) carbendazin + thiram + fipronil + polímero; 3 ) carbendazin + thiram + fipronil; 4) carbendazin + thiram + thiodicarb + polímero e 5) carbendazin + thiram + thiodicarb. Avaliaram-se emergência, eficiência no controle de lagarta elasmo (Elasmopalpus lignosellus) e sanidade das sementes. Nos resultados dos parâmetros emergência e controle de elasmo, os tratamentos associados ao inseticida fipronil foram superiores aos demais; na sanidade das sementes, os tratamentos com o inseticida thiodicarb foram mais eficientes, indicando ser esse o inseticida mais compatível à mistura com os fungicidas carbendazin + thiram aos 150 dias de armazenamento. Os produtos testados podem ser utilizados no tratamento de sementes de feijão, e as sementes tratadas armazenadas por até 120 dias antes da semeadura.
\end{abstract}

Palavras chaves: armazenamento de sementes, Elasmopalpus lignosellus, sanidade de sementes, testes fisiológicos.

\section{ABSTRACT \\ EFFECT OF THE STORAGE IN THE COMPATIBILITY OF FUNGICIDES AND INSECTICIDES ASSOCIATED OR NOT TO A POLYMER IN DRY BEAN SEED COATING}

The compatibility of chemical products in dry bean seed coating associated to a polymer was studied in this work. Also, the maximum period of storage for these seeds coated with polymer and defensives was established. Dry beans, from cv. Pérola were submitted to the following treatments: 1) control; 2) carbendazin + thiram + fipronil + polymer; 3 ) carbendazin + thiram + fipronil; 4) carbendazin + thiram + thiodicarb + polymer and 5) carbendazin + thiram + thiodicarb. The evaluated parameters included emergency, lesser cornstalk borer (Elasmopalpus lignosellus) efficiency control and seed pathology. For the parameters emergency and borer control the best results were obtained with the treatments including fipronil insecticide. As for seed pathology, treatments with thiodicarb insecticide were more efficient, indicating a suitable compatibility of this insecticide with the fungicides carbendazin + thiram until 150 days of storage. The products tested could be used in dry bean seed dressing, and the treated seeds could be stored up to hundred and twenty days prior to planting.

Key words: seed storage, Elasmopalpus lignosellus, seed pathology, physiological tests.

( ${ }^{1}$ ) Parte da Dissertação da primeira autora, apresentada à Universidade Federal de Goiás, Goiânia (GO). Recebido para publicação em 4 de maio de 2004 e aceito em 23 de maio de 2005.

$\left({ }^{2}\right)$ Doutoranda em Agronomia, Área de Produção Vegetal - Universidade Federal de Goiás, Caixa Postal 131, 74001-970 Goiânia (GO). E-mail: rosanagbarros@hotmail.com

$\left({ }^{3}\right)$ Embrapa Arroz e Feijão, Caixa Postal 179, 75375-000 Santo Antônio de Goiás (GO). E-mail: alex@cnpaf.embrapa.br

$\left({ }^{4}\right)$ Embrapa Tabuleiros Costeiros, Caixa Postal 44, 49001-970 Aracajú (SE). E-mail: jcosta@cpatc.embrapa.br

$\left({ }^{5}\right)$ Bolsista do CNPq. 


\section{INTRODUÇÃO}

A maioria das culturas destinadas à produção de alimentos está sujeita à incidência de pragas e doenças (NeERgARD, 1979), e a má qualidade das sementes representa uma das principais causas da baixa produtividade das lavouras de feijoeiro no Brasil (Costa e Silva e Silva, 1999; Freitas, 2001; Machado, 1986).

Por esse motivo, é crescente o interesse pelo tratamento químico das sementes, no qual se objetiva conferir-lhes proteção e às plântulas, delas originadas, contra a ação de patógenos e insetos-pragas. Assim, proporciona-se a manutenção da qualidade sanitária e fisiológica da semente, contribuindo para a obtenção do estande inicial almejado; além disso, reduz-se drasticamente a disseminação desses organismos nocivos na área (Dourado Neto e FANCELli, 2000).

Dentre os produtos empregados no tratamento químico das sementes, têm-se os fungicidas, inseticidas, herbicidas e reguladores de crescimento, que, conjuntamente, formam a bateria de agroquímicos disponíveis para proteção das culturas e manutenção de seu potencial de produção (Azevedo, 2001). Pouco se sabe sobre a compatibilidade da mistura desses produtos; no entanto, é sempre vantajoso que tenham características favoráveis à sua aplicação em mistura (MACHADO, 1988).

O tratamento químico de sementes, associado ao revestimento com polímeros, tem recebido especial atenção nos últimos anos (PIRES, 2000). O revestimento de sementes é um processo de aplicação de uma base fina e contínua, sólida ou líquida, contendo polímeros sólidos, dissolvidos ou em suspensão, que pode ser realizado juntamente com a incorporação de produtos fitossanitários, revestindo-se o seu tegumento natural (ScotT, 1989), de maneira que o microambiente de cada semente seja influenciado por esses materiais utilizados.

Dessa forma, buscou-se, neste trabalho, avaliar a compatibilidade de alguns produtos no tratamento de sementes de feijão em associação a um polímero e, também, estabelecer o período máximo de armazenamento possível para as sementes revestidas com polímero e defensivos.

\section{MATERIAL E MÉTODOS}

Nos experimentos foram utilizadas sementes de feijão (Phaseolus vulgaris L.), da cultivar Pérola, sendo necessários $10 \mathrm{~kg}$ em cada tratamento. Os tratamentos e as suas respectivas doses em $\mathrm{g}$ do ingrediente ativo $/ 100 \mathrm{~kg}$ de sementes consistiram de: 1) testemunha; 2 ) carbendazin + thiram + fipronil + polímero $-50+140+200+100 ; 3)$ carbendazin + thiram + fipronil $-50+140+200 ; 4)$ carbendazin + thiram + thiodicarb + polímero $-50+140+2000+$ 100 e 5) carbendazin + thiram + thiodicarb $-50+140$ + 2000. Logo após a aplicação do tratamento, as sementes foram secas à sombra e posteriormente, colocadas em sacos de aniagem e mantidas em armazém natural, sem controle da temperatura e umidade do ar, por um período de 150 dias.

Mensalmente e ao longo de todo o período de armazenamento - 0, 30, 60, 90, 120 e 150 dias, avaliaram-se os efeitos dos produtos usados na emergência e sanidade das sementes por meio dos seguintes testes:

\subsection{Testes Fisiológicos}

Teste de germinação (Germ-test)

O teste de germinação foi realizado de acordo com as RAS (BRASIL, 1992).

Emergência em casa de vegetação

Esse teste foi desenvolvido em casa de vegetação, com temperatura de $\pm 25^{\circ} \mathrm{C}$ e umidade relativa de $\pm 85 \%$. A unidade experimental foi composta de uma bandeja plástica, com capacidade para $2 \mathrm{~kg}$ de solo Latossolo Vermelho-Escuro, sendo distribuídas 200 sementes por tratamento, correspondendo a 50 sementes por bandeja. A profundidade de semeadura foi $2 \mathrm{~cm}$; as bandejas permaneceram em casa de vegetação até que as plântulas estivessem com 21 dias de idade, quando, então, determinou-se o estande referente ao número de plântulas normais emergidas em cada bandeja.

\section{Emergência em campo}

Esse teste foi realizado em canteiros contendo solo do mesmo tipo. Na semeadura, utilizaram-se 20 sementes por metro, formando-se parcelas de $3 \mathrm{~m}$ de comprimento, com três repetições por tratamento. Para o exame do efeito dos tratamentos nas sementes, determinou-se o estande de plântulas aos 15 dias após a semeadura, referente ao número de plântulas normais emergidas em cada canteiro.

\subsection{Controle de Lagarta Elasmo (Elasmopalpus lignosellus)}

Esse teste foi realizado para se avaliar a compatibilidade entre fungicidas e inseticidas 
utilizados no controle da lagarta elasmo, praga da fase inicial de desenvolvimento da cultura do feijoeiro.

O experimento foi desenvolvido em casa de vegetação, sendo cada unidade constituída de quatro vasos com cinco plântulas por tratamento. Para a realização desse teste, cada plântula originária das sementes submetidas aos diversos tratamentos foi infestada com uma larva neonata da broca do colo (Elasmopalpus lignosellus). A infestação foi realizada no $5 .^{\circ}$ dia após a emergência e a avaliação do experimento, aos 10 dias após a infestação, contandose a quantidade de plântulas atacadas pela lagarta elasmo.

Os dados, expressos em porcentagem de eficiência de controle, foram calculados pela fórmula de Henderson e Tilton (1955).

\subsection{Teste de Sanidade}

No método de incubação em papel filtro com congelamento, as sementes devidamente tratadas foram distribuídas em caixas de plástico transparente - gerbox, previamente desinfetadas com solução de álcool $70 \%$, forradas com duas folhas de papel de filtro esterilizadas e umedecidas com água destilada. A incubação foi realizada em câmara com temperatura controlada de $20 \pm 2{ }^{\circ} \mathrm{C}$, fotoperíodo de 12 horas de luz ultravioleta e 12 horas no escuro e, 24 horas depois, as sementes foram transferidas para o freezer. Transcorridas 24 horas, foram transferidas novamente para a câmara de incubação, onde permaneceram por sete dias. Foram utilizadas 400 sementes de feijão por tratamento, colocando-se 20 sementes/caixa de gerbox, totalizando-se 20 repetições. A contagem e identificação dos fungos foram feitas por meio de observações das colônias formadas ao redor e sobre as sementes, com auxílio de microscópio estereoscópio, e, quando necessário, do microscópio composto (BARNET e Hunter, 1972; MACHAdo, 1988; BRASIL, 1992).

\subsection{Procedimento estatístico}

Os experimentos foram instalados em delineamento experimental inteiramente casualizado, dentro de cada período de armazenamento $(0,30,60$, 90, 120 e 150 dias).

Para as análises estatísticas foram utilizadas as análises de variância e comparação de médias, pelo teste t. As variáveis emergência em campo, emergência em casa de vegetação e porcentagem de eficiência de controle da lagarta elasmo foram transformadas em arco seno $\sqrt{x / 100}$ e a variável sanidade (Rhizopus sp., Penicillium sp., Aspergillus sp., Fusarium sp.) em $\sqrt{x+1 / 2}$.

\section{RESULTADOS E DISCUSSÃO}

\subsection{Testes Fisiológicos}

Nos três métodos utilizados na avaliação da qualidade fisiológica das sementes - teste de germinação, emergência em casa de vegetação e emergência em campo, observou-se um declínio na porcentagem de plântulas emergidas, ao fim dos 150 dias de armazenamento (Tabela 1). Cardoso et al. (2004) também verificaram uma redução da qualidade fisiológica de sementes de soja tratadas com fungicidas, a partir do quarto mês de armazenamento.

\section{Teste de germinação}

Nesse método de avaliação, aos 0, 30 e 90 dias de armazenamento verificou-se maior porcentagem de germinação das sementes nos tratamentos com o inseticida fipronil - CTFP, CTF (Tabela 1). OliveIRA e CRUz (1986), em estudos com sementes de milho tratadas com inseticidas, verificaram efeito negativo do inseticida na germinação das sementes, sendo esse efeito intensificado com o prolongamento do período de armazenamento.

Aos 60 dias de armazenamento, todos os tratamentos utilizados foram superiores à testemunha na porcentagem de sementes germinadas, com exceção do tratamento thiodicarb sem o polímero (CTT) (Tabela 1).

Verificou-se também que, aos 60 e 120 dias de armazenamento, houve uma redução na porcentagem de germinação das sementes não tratadas (testemunha) quando comparadas às sementes tratadas. Essa redução na porcentagem de germinação pode ter ocorrido devido a fatores, tais como: erro de amostragem, teor de água nas sementes, falta de controle da temperatura e umidade do ar durante o armazenamento, dentre outros (Tabela 1).

Aos 150 dias de armazenamento, a testemunha e o tratamento fipronil sem o polímero CTF - foram os que mais se destacaram na porcentagem de sementes germinadas $(81,5 \%$ e $75 \%$, respectivamente).

\section{Emergência em casa de vegetação}

Não houve diferenças significativas entre os tratamentos, aos 0, 60 e 90 dias de armazenamento, na porcentagem de sementes germinadas (Tabela 1).

Nos tratamentos com o inseticida fipronil CTFP e CTF - aos 30 dias de armazenamento, verificouse maior porcentagem de sementes germinadas em relação aos tratamentos com o inseticida thiodicarb CTTP e CTT (Tabela 1). 
Tabela 1. Germinação de sementes e emergência de plântulas de feijoeiro (Phaseolus vulgaris L.) após tratamento das sementes com fungicidas, inseticidas e polímero, ao longo de 150 dias de armazenamento. Santo Antônio de Goiás (GO), 2001

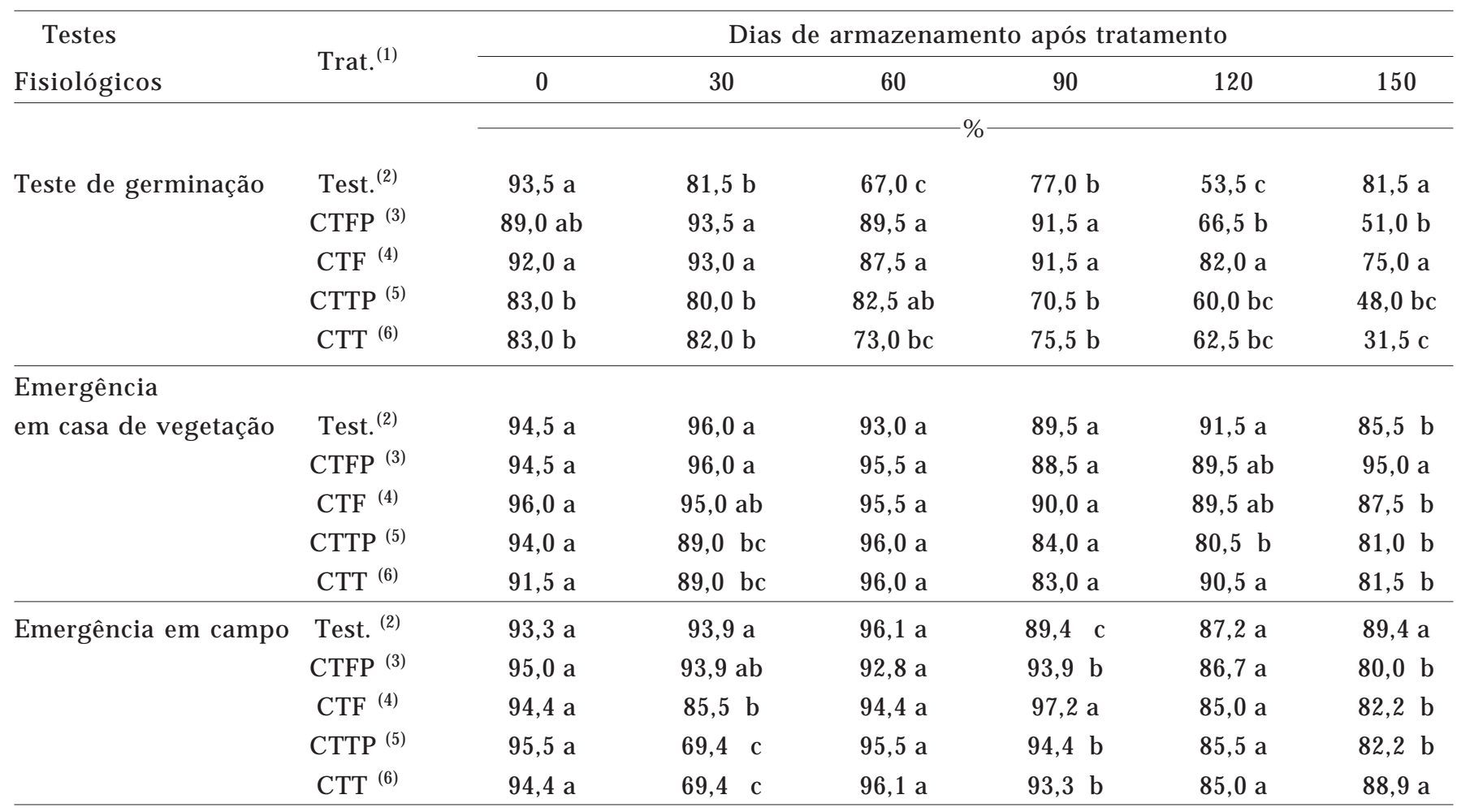

$\left({ }^{1}\right)$ Trat. $=$ Tratamentos; $\left({ }^{2}\right)$ Test. $=$ Sementes sem tratamento; $\left({ }^{3}\right)$ CTFP $=$ Sementes tratadas com Carbendazin, Thiram, Fipronil, Polímero; $\left({ }^{4}\right)$ $\mathrm{CTF}=$ Sementes tratadas com Carbendazin, Thiram, Fipronil; $\left({ }^{5}\right)$ CTTP = Sementes tratadas com Carbendazin, Thiram, Thiodicarb, Polímero; $\left({ }^{6}\right)$ CTT $=$ Sementes tratadas com Carbendazin, Thiram, Thiodicarb; Médias seguidas pela mesma letra, na coluna, não são estatisticamente diferentes entre si pelo teste $\mathrm{t}$, a $5 \%$ de probabilidade.

Aos 120 dias de armazenamento, no tratamento com o inseticida thiodicarb com polímero - CTTP - constatou-se redução de $10 \%$ na porcentagem de sementes germinadas em relação ao mesmo tratamento sem polímero - CTT (Tabela 1).

Aos 150 dias, o tratamento fipronil + polímero - CTFP - foi o único significativo quando comparado aos demais, com $95 \%$ de germinação. Com a presença do polímero no mesmo tratamento obteve-se um aumento de $9,5 \%$ na porcentagem de sementes germinadas em relação à testemunha (Tabela 1).

BARROS et al. (2001), estudando a compatibilidade do inseticida thiamethoxam com três fungicidas (difenoconazole, fludioxonil e carboxin) recomendados para o tratamento de sementes de feijão, verificaram que a emergência das plântulas em campo e casa de vegetação não foi afetada pelos tratamentos utilizados, visto que houve compatibilidade entre os produtos testados no controle da mosca-branca.

\section{Emergência em campo}

Aos 0, 60 e 120 dias de armazenamento, todos os tratamentos foram estatisticamente semelhantes na porcentagem de sementes germinadas (Tabela 1).
Aos 30 dias de armazenamento, nos tratamentos com o inseticida thiodicarb - CTTP e CTT - verificou-se uma redução na porcentagem de sementes germinadas, $24,5 \%$ respectivamente, em relação à testemunha (Tabela 1).

Observou-se que, aos 90 dias de armazenamento, todos os tratamentos utilizados foram superiores à testemunha na porcentagem de sementes germinadas. Com a presença do polímero no tratamento com fipronil - CTFP - contribuiu-se para uma redução de $3,3 \%$ no número de sementes germinadas em relação ao mesmo tratamento sem polímero - CTF (Tabela 1).

Aos 150 dias de armazenamento, nos tratamentos com o inseticida fipronil - CTFP e CTF e thiodicarb com polímero - CTTP - verificou-se redução na porcentagem de sementes germinadas - $9,4 \%, 7,2 \%$ e $7,2 \%$ - respectivamente, em relação à testemunha (Tabela 1).

Os dois inseticidas utilizados no tratamento das sementes foram compatíveis com os fungicidas, por não ter sido prejudicada a germinação das sementes (Tabela 1). 
Considerando os valores de cada tratamento ao longo do armazenamento, e excetuando-se CTTP e CTT aos 30 dias, nos testes de emergência não houve efeitos das misturas até aos 120 dias. Assim, o revestimento das sementes é uma técnica viável. PIRES et al. (2004) também verificaram que, na associação de polímeros ao tratamento químico com fungicidas não há interferência na germinação de sementes de feijão.

\subsection{Controle de Lagarta Elasmo (Elasmopalpus lignosellus)}

Com a presença do polímero nos tratamentos com fipronil e thiodicarb - CTFP e CTTP, ao zero dia de armazenamento, constatou-se uma redução de $23,53 \%$ e $29,42 \%$, respectivamente, na porcentagem de controle da praga quando comparados aos mesmos tratamentos sem polímero - CTF e CTT (Tabela 2).

Aos 60 dias de armazenamento das sementes, não houve diferença estatística entre os tratamentos utilizados no controle da praga. Já aos 90 dias, no tratamento com o inseticida fipronil sem polímero CTF, verificou-se eficiência de $90 \%$ no controle da praga (Tabela 2).

Aos 30 e 120 dias verificou-se eficácia nos tratamentos com o inseticida fipronil sem polímero CTF e thiodicarb - CTTP e CTT no controle da lagarta elasmo (Tabela 2).

Aos 150 dias de armazenamento, verificou-se eficiência nos tratamentos com o inseticida fipronil CTFP e CTF - no controle da lagarta elasmo. Com a presença do polímero no tratamento com fipronil CTFP - contribuiu-se para um aumento no controle da praga em relação ao tratamento com o thiodicarb, com o qual no fim desse período, obteve-se controle de apenas $47,37 \%$ da praga (Tabela 2 ).

Comparando-se os inseticidas fipronil e thiodicarb no controle da lagarta elasmo, no fim do período de armazenamento, verificou-se superioridade do fipronil em relação ao thiodicarb (Tabela 2).

\subsection{Teste de Sanidade}

Os fungos detectados nas sementes de feijão durante os 150 dias de armazenamento foram Rhizopus sp., Penicillium sp., Aspergillus sp. e Fusarium solani f. sp. phaseoli, de ocorrência natural no lote de sementes utilizado (Tabela 3 ).

O Rhizopus sp. foi o organismo com maior incidência nas sementes, sendo de 9,7\% o número de sementes contaminadas, ao longo dos 150 dias de armazenamento. Dentre os tratamentos utilizados, os únicos com eficiência na redução da porcentagem de sementes contaminadas por Rhizopus sp., ao longo dos 150 dias de monitoramento, foram aqueles com thiodicarb CTTP e CTT - sendo eliminado praticamente todo o fungo presente nas sementes. A porcentagem de sementes contaminadas por Rhizopus sp., no início do armazenamento, foi bem menor do que aquela ao longo deste período (Tabela 3). Resultados similares foram obtidos por CARDOSO et al. (2001), em sementes de soja.

Tabela 2. Porcentagem de controle da lagarta elasmo em plântulas de feijoeiro (Phaseolus vulgaris L.), após tratamento de sementes com fungicidas, inseticidas e polímero, ao longo de 150 dias de armazenamento. Santo Antônio de Goiás (GO), 2001

\begin{tabular}{|c|c|c|c|c|c|c|}
\hline \multirow{2}{*}{ Tratamentos } & \multicolumn{6}{|c|}{ Dias de armazenamento após tratamento } \\
\hline & 0 & 30 & 60 & 90 & 120 & 150 \\
\hline & & & 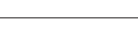 & 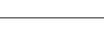 & & \\
\hline Test. ${ }^{(1)}$ & $0 \mathrm{a}$ & 0 a & $0 \mathrm{a}$ & 0 a & $0 \mathrm{a}$ & $0 \mathrm{a}$ \\
\hline CTFP (2) & 70,59 ba & $75 \mathrm{~b}$ & $89,47 \mathrm{~b}$ & $85 \mathrm{bc}$ & $68,42 \mathrm{~b}$ & $78,95 \mathrm{~cd}$ \\
\hline $\mathrm{CTF}^{(3)}$ & $94,12 \mathrm{c}$ & $85 \mathrm{bc}$ & $89,47 \mathrm{~b}$ & $90 \mathrm{c}$ & $73,68 \mathrm{bc}$ & $84,21 \mathrm{~d}$ \\
\hline СТTP ${ }^{(4)}$ & $64,70 \mathrm{~b}$ & $85 \mathrm{bc}$ & $94,74 \mathrm{~b}$ & $85 \mathrm{bc}$ & $73,68 \mathrm{bc}$ & $47,37 \mathrm{~b}$ \\
\hline $\mathrm{CTT}^{(5)}$ & $94,12 \mathrm{c}$ & $95 \mathrm{c}$ & $89,47 \mathrm{~b}$ & $70 \mathrm{~b}$ & $89,47 \mathrm{c}$ & 57,89 bc \\
\hline
\end{tabular}

$\left({ }^{1}\right)$ Test $=$ Sementes sem tratamento; $\left({ }^{2}\right) \mathrm{CTFP}=$ Sementes tratadas com Carbendazin, Thiram, Fipronil, Polímero; $\left({ }^{3}\right) \mathrm{CTF}=\mathrm{Sementes}$ tratadas com Carbendazin, Thiram, Fipronil; $\left({ }^{4}\right)$ CTTP $=$ Sementes tratadas com Carbendazin, Thiram, Thiodicarb, Polímero; ${ }^{5} \mathrm{CTT}=\mathrm{Sementes}$ tratadas com Carbendazin, Thiram, Thiodicarb; Médias seguidas pela mesma letra, na coluna, não são estatisticamente diferentes entre si pelo teste t, a $5 \%$ de probabilidade. 
Tabela 3. Incidência percentual de microrganismos em sementes de feijão (Phaseolus vulgaris L.) tratadas com fungicidas, inseticidas e polímero, ao longo de 150 dias de armazenamento. Santo Antônio de Goiás (GO), 2001

\begin{tabular}{|c|c|c|c|c|c|c|c|}
\hline \multirow{2}{*}{ Fungos } & \multirow{2}{*}{ Trat. ${ }^{(1)}$} & \multicolumn{6}{|c|}{ Dias de armazenamento após tratamento } \\
\hline & & 0 & 30 & 60 & 90 & 120 & 150 \\
\hline & & & & 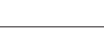 & & & \\
\hline \multirow[t]{5}{*}{ Rhizopus } & Test. ${ }^{(2)}$ & $2,4 \mathrm{a}$ & $0,3 \mathrm{ab}$ & $6,3 \mathrm{a}$ & $2,2 \mathrm{a}$ & $2,1 \mathrm{ab}$ & $9,7 \mathrm{a}$ \\
\hline & CTFP ${ }^{(3)}$ & $0,8 \mathrm{ab}$ & $1,0 \mathrm{a}$ & $2,9 \mathrm{~b}$ & $0,4 \mathrm{a}$ & $3,2 \mathrm{a}$ & $4,6 \mathrm{~b}$ \\
\hline & $\mathrm{CTF}^{(4)}$ & $0,1 \mathrm{~b}$ & $1,3 \mathrm{a}$ & 0,1 c & $0 \mathrm{a}$ & $2,1 \mathrm{ab}$ & $5,1 \mathrm{~b}$ \\
\hline & СТTP ${ }^{(5)}$ & $0 \mathrm{~b}$ & $0 \mathrm{~b}$ & $0,1 \quad c$ & $0 \mathrm{a}$ & $0,6 \mathrm{~b}$ & $0,4 \quad \mathrm{c}$ \\
\hline & $\mathrm{CTT}^{(6)}$ & $0 \mathrm{~b}$ & $0 \mathrm{~b}$ & $0,1 \mathrm{c}$ & $1,0 \mathrm{a}$ & $0,5 \mathrm{~b}$ & $0 \mathrm{c}$ \\
\hline \multirow[t]{5}{*}{ Penicillium } & Test. ${ }^{(2)}$ & $0,7 \mathrm{a}$ & $1,5 \mathrm{a}$ & $0,5 \mathrm{a}$ & $2,9 \mathrm{a}$ & $1,6 \mathrm{a}$ & $0,6 \mathrm{a}$ \\
\hline & $\mathrm{CTFP}^{(3)}$ & $0,3 \mathrm{~b}$ & $0 \mathrm{~b}$ & $0 \mathrm{~b}$ & $0 \mathrm{~b}$ & $0,1 \mathrm{~b}$ & $0 \mathrm{~b}$ \\
\hline & $\mathrm{CTF}^{(4)}$ & $0 \mathrm{~b}$ & $0 \mathrm{~b}$ & $0 \mathrm{~b}$ & $0 \mathrm{~b}$ & $0,1 \mathrm{~b}$ & $0 \mathrm{~b}$ \\
\hline & СТTP ${ }^{(5)}$ & $0 \mathrm{~b}$ & $0 \mathrm{~b}$ & $0 \mathrm{~b}$ & $0 \mathrm{~b}$ & $0 \mathrm{~b}$ & $0 \mathrm{~b}$ \\
\hline & $\mathrm{CTT}^{(6)}$ & $0 \mathrm{~b}$ & $0 \mathrm{~b}$ & $0 \mathrm{~b}$ & $0 \mathrm{~b}$ & $0 \mathrm{~b}$ & $0 \mathrm{~b}$ \\
\hline \multirow[t]{5}{*}{ Aspergillus } & Test. $^{(2)}$ & $0,6 \mathrm{a}$ & $0,8 \mathrm{a}$ & $1,0 \mathrm{a}$ & $1,4 \mathrm{a}$ & $0,8 \mathrm{a}$ & $2,8 \mathrm{a}$ \\
\hline & CTFP (3) & $0,1 \mathrm{~b}$ & $0 \mathrm{~b}$ & $0 \mathrm{~b}$ & $0 \mathrm{~b}$ & $0 \mathrm{~b}$ & $0 \mathrm{~b}$ \\
\hline & $\mathrm{CTF}^{(4)}$ & $0 \mathrm{~b}$ & $0 \mathrm{~b}$ & $0 \mathrm{~b}$ & $0 \mathrm{~b}$ & $0 \mathrm{~b}$ & $0 \mathrm{~b}$ \\
\hline & СТTP ${ }^{(5)}$ & $0 \mathrm{~b}$ & $0 \mathrm{~b}$ & $0 \mathrm{~b}$ & $0 \mathrm{~b}$ & $0 \mathrm{~b}$ & $0 \mathrm{~b}$ \\
\hline & $\mathrm{CTT}^{(6)}$ & $0 \mathrm{~b}$ & $0 \mathrm{~b}$ & $0 \mathrm{~b}$ & $0 \mathrm{~b}$ & $0 \mathrm{~b}$ & $0 \mathrm{~b}$ \\
\hline \multirow[t]{5}{*}{ Fusarium } & Test. (2) & $0,4 \mathrm{a}$ & $0,5 \mathrm{a}$ & $0,5 \mathrm{a}$ & $0 \mathrm{a}$ & $0 \mathrm{a}$ & $0 \mathrm{a}$ \\
\hline & $\mathrm{CTFP}^{(3)}$ & $0,1 \mathrm{ab}$ & $0,1 \mathrm{~b}$ & $0,2 \mathrm{a}$ & $0 \mathrm{a}$ & $0 \mathrm{a}$ & $0 \mathrm{a}$ \\
\hline & $\mathrm{CTF}^{(4)}$ & $0 \mathrm{~b}$ & $0 \mathrm{~b}$ & $0,1 \mathrm{a}$ & $0 \mathrm{a}$ & $0 \mathrm{a}$ & $0 \mathrm{a}$ \\
\hline & $\mathrm{CTTP}^{(5)}$ & $0 \mathrm{~b}$ & $0 \mathrm{~b}$ & $0 \mathrm{a}$ & $0 \mathrm{a}$ & $0,1 \mathrm{a}$ & $0 \mathrm{a}$ \\
\hline & $\mathrm{CTT}^{(6)}$ & $0 \mathrm{~b}$ & $0 \mathrm{~b}$ & 0 a & $0 \mathrm{a}$ & $0 \mathrm{a}$ & $0 \mathrm{a}$ \\
\hline
\end{tabular}

$\left({ }^{1}\right)$ Trat. $=$ Tratamentos; $\left({ }^{2}\right)$ Test $=$ Sementes sem tratamento; $\left({ }^{3}\right)$ CTFP $=$ Sementes tratadas com Carbendazin, Thiram, Fipronil, Polímero; $\left({ }^{4}\right)$ $\mathrm{CTF}=$ Sementes tratadas com Carbendazin, Thiram, Fipronil; $\left({ }^{5}\right) \mathrm{CTTP}=$ Sementes tratadas com Carbendazin, Thiram, Thiodicarb, Polímero; $\left({ }^{6}\right)$ CTT $=$ Sementes tratadas com Carbendazin, Thiram, Thiodicarb; Médias seguidas pela mesma letra, na coluna, não são estatisticamente diferentes entre si pelo teste $t$, a $5 \%$ de probabilidade.

Todos os tratamentos foram eficientes no controle dos fungos de armazenamento Penicillium sp. e Aspergillus sp., sendo possível a eliminação desses fungos das sementes ao longo de todo o período de armazenamento (Tabela 3). Goulart (1998) também obteve resultados similares em sementes de soja, erradicando-se esses mesmos fungos presentes nas sementes com a mistura carbendazin + thiram.

O fungo Fusarium solani f. sp. phaseoli esteve presente nas sementes até os 60 dias de armazenamento. Após esse período, o patógeno não foi mais detectado, o que pode ser devido ao aumento na porcentagem de fungos de armazenamento, principalmente Rhizopus sp., sendo mascarada, assim, sua detecção pelo método utilizado (Tabela 3). Resultados similares foram obtidos por PIRES et al. (2004) em sementes de feijão.
Nesse teste de sanidade, obteve-se melhor controle efetivo dos fungos presentes nas sementes com o inseticida thiodicarb do que com o inseticida fipronil, quando associado aos fungicidas carbendazin + thiram.

A necessidade de testes com maior número de fungicidas e inseticidas, utilizando lotes de sementes com diferentes níveis de danos, seria desejável para validar ou auxiliar no posicionamento dessa tecnologia em campo.

Trabalhos dessa natureza são, portanto, extremamente recomendáveis, pois a eficiência de uma tecnologia pode ser afetada por outras já existentes, principalmente quando utilizam o mesmo meio para a veiculação, que neste caso foi a semente. Um bom exemplo desse princípio são os trabalhos de BABU et 
al. (1993), em arroz, em que a compatibilidade de três inseticidas (monocrotophos, quinalphos e $\mathrm{HCH}$ ) e três fungicidas (captafol, edifenphos e carbendazin) foram testados contra pragas e doenças, utilizando Nilaparvata lugens e Rhizoctonia solani como modelo biológico. No trabalho mencionado, verificou-se que não houve sinergismo na atividade dos inseticidas para doses baixas de fungicidas. Contudo, ocorreu efeito contrário quando doses mais elevadas dos fungicidas foram empregadas, comprometendo a eficiência dos inseticidas.

\section{CONCLUSÕES}

1) Há decréscimo na qualidade fisiológica das sementes de feijão tratadas com defensivos e revestidas com o polímero ao longo dos 150 dias de armazenamento;

2) Há compatibilidade entre os inseticidas fipronil e thiodicarb e os fungicidas carbendazin sem prejuízo à germinação das sementes;

3) Há maior compatibilidade entre o inseticida fipronil e a mistura carbendazin + thiram no controle da lagarta elasmo até aos 150 dias de armazenamento e entre os fungicidas carbendazin e thiram e o inseticida thiodicarb no controle efetivo de fungos nas sementes.

\section{REFERÊNCIAS}

AZEVEDO, L.A.S. Paradigmas da proteção de plantas com fungicidas. In: AZEVEDO, L.A.S. (Ed.). Proteção integrada de plantas com fungicidas. 1.ed. São Paulo, 2001. p.151-160.

BABU, K.; REGHUNATH, P.; MOHANDAS, N.; WILSON, K.I. Compatibility of insecticides and fungicides on mortality of Nilaparvata lugens (Stall) and on the inhibition of Rhizoctonia solani Kuhn. CAB Abstracts [on line]. India, v.31, n.1, p.86-90. 1993. Disponível em: http:/ / www.cdrompro.com.br/cgi-bin/ wsufg_agrivet.cgi Acesso em: 21 mar. 2005.

BARNET, H.L.; HUNTER, B.B. Illustrate genera of imperfect fungi. 3.ed. Minneapolis: Burges, 1972. 241p.

BARROS, R.G.; YOKOYAMA, M.; COSTA, J.L.S. Compatibilidade do inseticida thiamethoxam com fungicidas utilizados no tratamento de sementes de feijoeiro. Pesquisa Agropecuária Tropical, Goiânia, v.21, n.2, p.153-157, 2001.

BRASIL. Regras para Análise de Sementes. Brasília: Ministério da Agricultura e da Reforma Agrária / Secretaria Nacional de Defesa Agropecuária, 1992. 365p.

CARDOSO, P.C.; BAUDET, L.; PESKE, S.T. Armazenamento em sistema a frio de sementes de soja tratadas com fungicida. Revista Brasileira de Sementes, Brasília, 2004, v.26, n.1, p.15-23.
CARDOSO, P.C.; BAUDET, L.M.; LUCCA-FILHO, O.A. Sanidade de sementes de soja tratadas com fungicida, armazenadas em sistema a frio. Informativo Abrates, Londrina, v.11, n.2. 2001. (Trabalho, 246).

COSTA, J.L.S.; SILVA, M.B. Tratamento de sementes. In: CANTERI, M.G.; PRIA, M.D.; SILVA, O.C. (Eds.). Principais doenças fúngicas do feijoeiro: orientações para manejo econômico e ecológico. Ponta Grossa: Editora: UEPG, 1999. p.111-125.

DOURADO NETO, D.; FANCELLI, A.L. Implantação da cultura In: DOURADO NETO, D.; FANCELLI, A.L. (Eds.). Produção de feijão. Guaíba: Agropecuária, 2000. p. 87-133.

FREITAS, R.A. (Coord.). Patologia de sementes de feijão. Disponível em: http:/ /orbita.starmedia.com/ fitopatologia/ patofeijao.htm. Acesso em 2 ago. 2001.

GOULART, A.C.P. Eficiência do tratamento de sementes de soja com fungicidas visando o controle de patógenos. Dourados: Embrapa CPAO, 1998. 20p. (Boletim de Pesquisa, 4)

HENDERSON, C.F.; TILTON, E.H. Tests with acaricides against the drown wheat white. Journal of Economic Entomology, Lanham, v.48, n.1. 1955. p.157-161.

MACHADO, J.C. Tratamento de Sementes de Feijão. In: SIMPÓSIO BRASILEIRO DE PATOLOGIA DE SEMENTES, 2. , 1986. Campinas. Anais... Campinas: Fundação Cargill, 1986. p.131-37.

MACHADO, J.C. Patologia de sementes: fundamentos e aplicações. Lavras: ESAL/FAEPE, 1988. 107p.

NEERGAARD, P. Seed Pathology. London: The Mac Millan Press, 1979. 839p.

OLIVEIRA, L.J.; CRUZ, I. Efeito de diferentes inseticidas e dosagens na germinação de sementes de milho (Zea mays L.). Pesquisa Agropecuária Brasileira, Brasilia, v.21, n.6, p.578-585, 1986.

PIRES, L.L. Efeito do revestimento com polímeros na fixação e na ação de fungicidas à semente de feijão (Phaseolus vulgaris L.). 2000. 120f. Dissertação (Mestrado em Produção Vegetal) Escola de Agronomia e Engenharia de Alimentos Universidade Federal de Goiás, Goiânia.

PIRES, L.L.; BRAGANTINI, C.; COSTA, J.L.S. Armazenamento de sementes de feijão revestidas com polímeros e tratadas com fungicidas. Pesquisa Agropecuária Brasileira, Brasília, v.39, n.7, p.709-715, 2004.

SCOTT, J.M. Seed coatings and treatments and their effects on plant establishment. Advances in Agronomy, New York, v.42, p.43-83, 1989. 